\title{
Benzotriazole and Tolytriazole as Corrosion Inhibitors of Carbon Steel 1008 in Sulfuric Acid
}

\author{
Douglas K. da Silva, ${ }^{\text {a,b) }}$ Gisele C.B. Ribas, ${ }^{\text {b) }}$ Maico T. da Cunha, ${ }^{\text {a,b) }}$ \\ Silvia M.L. Agostinho, ${ }^{\text {b) }}$ Paulo R.P. Rodrigues ${ }^{\text {a) * }}$ \\ ${ }^{a)}$ GPEL - Electrochemical Research Group of DEQ / UNICENTRO - Department of Chemistry \\ / State University of the Center-West, Guarapuava - Paraná, Brazil

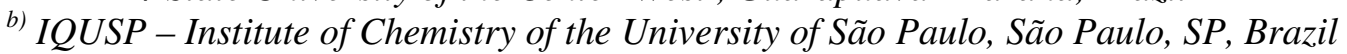

18 June 2004; Accepted 19 June 2006

\begin{abstract}
In this work the characteristics of corrosion and inhibition of 1008 stainless steel in 0.5 mol. $\mathrm{L}^{-1} \mathrm{H}_{2} \mathrm{SO}_{4}$ were investigated by physical and electrochemical methods. Organic compounds containing the heteroatom of $\mathrm{N}$ as benzotriazole (BTAH), tolytriazole (TTAH) and mixtures of BTAH + TTAH were used as corrosion inhibitors. The techniques used were: open circuit potential, anodic potentiodynamic polarization measurements, chronoamperometry, electrochemical impedance spectroscopy, weight loss and optical microscopy. The anodic polarization showed that the increase of both concentrations BTAH or TTAH diminishes the current density in all the potential anodic range studied; these results together with the weight loss measurements suggest that the inhibitors act by blocking the surface and that the interaction among them is only of destructive nature when inhibiting efficiency is approximated $100 \%$, revealing that at low concentration both inhibitors act without affecting the mechanisms of the cathodic processes, but the anodic polarization curves in presence of the TTAH showed a strong inhibitive effect in all the potential range studied. However, both gravimetric and electrochemical results suggest a synergic effect for the inhibitive efficiency of the mixture $1.10^{-3}$ mol.L $L^{-1}$ BTAH $+1.10^{-3}$ mol. $\mathrm{L}^{-1}$ TTAH.
\end{abstract}

Keywords: carbon steel 1008, corrosion, inhibitor, sulfuric acid, benzotriazole, tolytriazole.

\section{Introdução}

Compostos orgânicos contendo heteroátomos, como nitrogênio e enxofre são usualmente empregados como inibidores de corrosão metálica $[1,2,3]$. Compostos heterocíclicos, triazólicos, em particular, o benzotriazol (BTAH) e o tolitriazol (TTAH), vêm sendo investigados como inibidores de corrosão para materiais ferrosos [4-10].

Nobe [7] estudou o efeito de compostos triazólicos na interface $\mathrm{Fe} / \mathrm{H}^{+}$. Foi mostrado, a partir de curvas de polarização, que o BTAH e o TTAH inibem a

\footnotetext{
* Corresponding author. E-mail address: prprodrigues@unicentro.br
} 
reação $\mathrm{H}^{+} / \mathrm{H}_{2}$ sobre substrato de ferro, em meio de $\mathrm{H}_{2} \mathrm{SO}_{4} 0.5$ mol.L $\mathrm{L}^{-1}$, embora não afetem, o mecanismo da referida reação. Os estudos de polarização anódica mostraram que a eficiência inibidora depende da concentração: o BTAH é melhor inibidor do que o TTAH a concentração mais elevada (20 mmol.L $\left.{ }^{-1}\right)$, enquanto a baixas concentrações $\left(0.1 \mathrm{mmol} . \mathrm{L}^{-1}\right)$ o comportamento se inverte. Estudos realizados por Rodrigues [4] mostraram que o comportamento do BTAH para a interfase aço carbono / ácido sulfúrico despende da concentração deste ácido: uma solução contendo $1.0 \mathrm{mmol} . \mathrm{L}^{-1}$ de BTAH exerce efeito catalisador em meio de $\mathrm{H}_{2} \mathrm{SO}_{4} 2.0$ mol.L ${ }^{-1}$, enquanto a sua ação inibidora é notável em $\mathrm{H}_{2} \mathrm{SO}_{4} 0.5$ mol.L ${ }^{-1}$. O efeito do BTAH, em diferentes concentrações, na corrosão do aço baixo carbono em meio de $\mathrm{H}_{2} \mathrm{SO}_{4} 1.0$ mol.L $\mathrm{L}^{-1}$ foi estudado por Gomma [10], empregando polarização potenciodinâmica a baixa velocidade de varredura. A máxima eficiência inibidora igual a $98.5 \%$ foi obtida para BTAH $9 \times 10^{-3}$ mol.L . $^{-1}$ Santos [6] verificou que a ação inibidora do BTAH para aço carbono em meio de $\mathrm{H}_{2} \mathrm{SO}_{4}$ não ultrapassa $98 \%$, em virtude da presença de inclusões neste material. Este trabalho tem como objetivo estudar a ação do BTAH, do TTAH e de misturas BTAH-TTAH como inibidores de corrosão para o aço carbono 1008 em meio de $\mathrm{H}_{2} \mathrm{SO}_{4} 0.5$ mol. $\mathrm{L}^{-1}$.

\section{Parte experimental}

\section{Materiais}

O benzotriazol, o tolitriazol e o ácido sulfúrico empregados foram de grau analítico.

A composição química das amostras de aço carbono ABNT 1008 estudadas é apresentada na Tabela 1. As análises químicas da composição elementar do aço carbono ABNT 1008, foram executadas com espectrômetro de emissão atômica marca Spectro e através de técnicas de microanálise.

Tabela 1. Composição química do aço carbono 1008.

\begin{tabular}{|l|c|c|c|c|c|}
\hline Elemento & $\mathrm{C}$ & $\mathrm{Mn}$ & $\mathrm{P}$ & $\mathrm{S}$ & $\mathrm{Fe}$ \\
\hline$\% \mathrm{~m} / \mathrm{m}$ & 0.08 & 0.330 & 0.048 & 0.021 & 99.52 \\
\hline
\end{tabular}

\section{Medidas gravimétricas}

Para os ensaios gravimétricos as amostras de aço carbono ABNT 1008 apresentavam uma área média de $25 \mathrm{~cm}^{2}$. As amostras foram previamente lixadas com lixas de $\mathrm{SiC}$ (carbeto de silício) de grana 320, 400 e 600, sucessivamente, lavadas com água e etanol e secas a ar quente. Todos os experimentos foram repetidos pelo menos 3 vezes.

\section{Análise morfológica da superfície}

As análises da superfície foram feitas empregando um microscópio óptico, modelo Olympus BX41M e um microscópio eletrônico de varredura (MEV) modelo XL-30 da marca Philips.

Nas análises ópticas o polimento prévio das amostras de aço carbono 1008 foi executado utilizando pasta de diamante de $1 \mu \mathrm{m}$. 


\section{Estudos eletroquímicos}

Nos estudos eletroquímicos, a área do eletrodo de trabalho de aço 1008 era de 1 $\mathrm{cm}^{2}$. Foram utilizados como eletrodos de referência o eletrodo de sulfato mercuroso (ESM), e como auxiliar o de platina, de área média de $20 \mathrm{~cm}^{2}$.

Nas medidas potenciostáticas anódicas e catódicas empregou-se um potenciostato desenvolvido pelo grupo que é descrito anteriormente [11] e nos ensaios de impedância eletroquímica empregou-se um potenciostato / galvanostato / frequencímetro da Gamry, modelo PC4/EIS 300, acoplado a um microcomputador para aquisição de dados. Todas as curvas de polarização foram levantadas a partir do potencial de corrosão $\left(\mathrm{E}_{\text {corr }}\right)$, isto é, o potencial de circuito aberto "quase" estacionário.

Para obtenção dos diagramas de impedância aguardou-se a estabilização do $\mathrm{E}_{\text {corr }}$ e aplicou-se um potencial de - $900 \mathrm{mV} / \mathrm{ESM}$. Esperou-se a estabilização da densidade de corrente neste potencial aplicado e executou-se uma perturbação de $+5 \mathrm{mV}$.

Os voltímetros empregados nas medidas de circuito aberto e potenciostáticas eram de 4 1/2 dígitos da Minipa modelo ET-2200.

\section{Temperatura de trabalho}

Todos os experimentos foram feitos a temperatura ambiente de $(19 \pm 1)^{\circ} \mathrm{C}$.

\section{Resultados e discussão}

\section{Ensaios gravimétricos}

Os resultados de perda de massa foram convertidos na forma de velocidade de corrosão $\left(\mathrm{v}_{\text {corr }}\right)$ de acordo com a equação I

$$
\mathrm{v}_{\text {corr }}=(\Delta \mathrm{m} / \mathrm{A} . \Delta \mathrm{t})
$$

onde $\mathrm{v}_{\text {corr }}=$ velocidade de corrosão, em g.cm $\mathrm{cm}^{-2} \cdot \mathrm{s}^{-1} ; \mathrm{A}=$ área da amostra de aço carbono $1008, \mathrm{em} \mathrm{cm}^{-2} ; \Delta \mathrm{t}=$ intervalo de tempo de imersão da amostra de aço carbono $1008 \mathrm{em}$ segundos (s).

Os resultados para a imersão em meio de $\mathrm{H}_{2} \mathrm{SO}_{4} 0.5$ mol.L $\mathrm{L}^{-1}$, na ausência e presença de BTAH ou TTAH e das misturas BTAH + TTAH são apresentados na Tabela 2 na forma de eficiência inibidora. Estes valores correspondem a uma corrosão generalizada, tanto na ausência quanto na presença de BTAH e/ou TTAH, o que foi constatado pelos valores constante de velocidade de corrosão $\left(\mathrm{v}_{\text {corr }}\right)$ num intervalo de tempo compreendido entre 30 minutos e 2 horas.

A eficiência inibidora $(\theta)$ foi calculada de acordo com a equação II:

$$
\theta=\left[\left(\mathrm{v}_{\text {corr }}-\mathrm{v}_{\text {corr (I) }}\right) / \mathrm{v}_{\text {corr }}\right] .100
$$

onde: $\theta$ = eficiência inibidora do composto orgânico; $\mathrm{v}_{\mathrm{corr}}=$ velocidade de corrosão na ausência do composto orgânico, em g. $\mathrm{cm}^{-2} \cdot \mathrm{s}^{-1} ; \mathrm{v}_{\mathrm{corr}(\mathrm{I})}=$ velocidade de corrosão na presença do composto orgânico, em g.cm $\mathrm{cm}^{-2} \cdot \mathrm{s}^{-1}$. 
Tabela 2. Eficiência inibidora (\%) obtida a partir dos ensaios gravimétricos para o aço carbono ABNT 1008 em meio de $\mathrm{H}_{2} \mathrm{SO}_{4} 0.5$ mol. $\mathrm{L}^{-1}$, na presença de BTAH, TTAH e BTAH + TTAH.

\begin{tabular}{cccc}
\hline & \multicolumn{3}{c}{$[\mathrm{BTAH}] / \mathrm{mol}^{-\mathrm{L}^{-1}}$} \\
{$[\mathrm{TTAH}] / \mathrm{mol} . \mathrm{L}^{-1}$} & 0 & $33 \pm 2$ & $1 \times 10^{-2}$ \\
\hline 0 & 0 & $74 \pm 3$ & $80 \pm 2$ \\
$1 \times 10^{-3}$ & $42 \pm 2$ & $96 \pm 2$ & $94 \pm 5$ \\
$1 \times 10^{-2}$ & $94 \pm 4$ & $88 \pm 2$ \\
\hline
\end{tabular}

Através da Tabela 2, se verifica, para todas as concentrações estudadas de BTAH e TTAH, a ação inibidora destes compostos para o aço carbono ABNT 1008 em meio de $\mathrm{H}_{2} \mathrm{SO}_{4} 0.5$ mol. $\mathrm{L}^{-1}$. Ressalta-se o efeito aditivo na ação inibidora para misturas de BTAH $1 \times 10^{-3}$ mol.L $\mathrm{L}^{-1}+$ TTAH $1 \times 10^{-3}$ mol.L $\mathrm{L}^{-1}(\theta=74 \%)$ quando comparada à ação apenas do BTAH (33\%) e do TTAH (42\%) na mesma concentração. Observa-se, por outro lado, que nas duas concentrações estudadas $\left(1 \times 10^{-2}\right.$ mol.L ${ }^{-1}$ e $1 \times 10^{-3}$ mol.L $\left.{ }^{-1}\right)$ o TTAH é mais eficiente que o BTAH. Nota-se ainda que para concentrações iguais a $1 \times 10^{-2}$ mol.L $\mathrm{L}^{-1}$ a mistura TTAH-BTAH se apresentou com menor eficiência do que os inibidores separadamente.

\section{Análise morfológica da superfície, por microscopia óptica}

A Fig. 1 corresponde à microscopia do aço carbono 1008 após polimento até pasta diâmetro de $1 \mu \mathrm{m}$. Observam-se inclusões alveolares e alongadas.

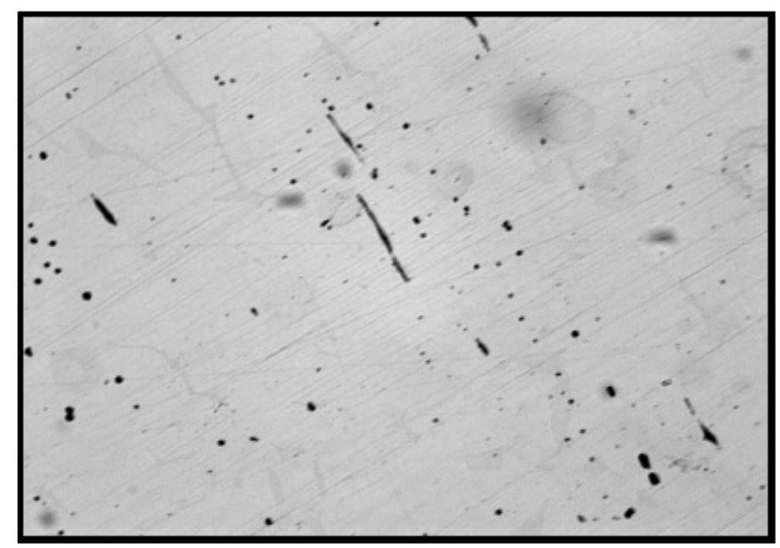

Figura 1. Micrografia óptica do aço carbono 1008 após polimento até pasta diamante de $1 \mu \mathrm{m}$. Aumento de $1000 \times$.

Na Tabela 3 são apresentadas a área média examinada, a área média de inclusões e a percentagem de inclusões para um total de seis análises realizadas. Observase uma média de $(1.6 \pm 0.4) \%$ de área superficial ocupada por inclusões.

Tabela 3. Análise quantitativa do percentual de número de inclusões presentes em uma área média da superfície da amostra do aço carbono 1008.

\begin{tabular}{|c|c|c|c|}
\hline Número de análises & $\begin{array}{c}\text { Área média } \\
\text { examinada }(1) / \mu \mathrm{m}^{2}\end{array}$ & $\begin{array}{c}\text { Área média de } \\
\text { inclusões }(2) / \mu \mathrm{m}^{2}\end{array}$ & $\begin{array}{c}\text { Relação: } \\
\{(2) /(1)\}^{*} 100\end{array}$ \\
\hline 06 & 107352.1 & $1741.99 \pm 413.21$ & $1.6 \pm 0.4$ \\
\hline
\end{tabular}


Análise morfológica da superfície, por microscopia eletrônica de varredura Nas Fig. 2.1 a 2.6 são apresentadas as micrografias do aço carbono 1008 após imersão em $\mathrm{H}_{2} \mathrm{SO}_{4} 0.5$ mol.L ${ }^{-1}$ na ausência e presença de BTAH e de TTAH nas duas concentrações estudadas.

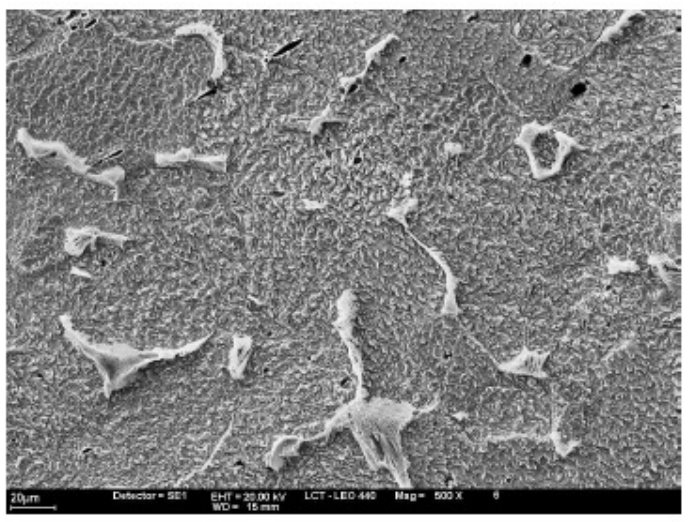

Figura 2.1. Aço 1008 atacado por $\mathrm{H}_{2} \mathrm{SO}_{4} 0.5$ mol. $L^{-1}$ na ausência de inibidores $-500 \times$.

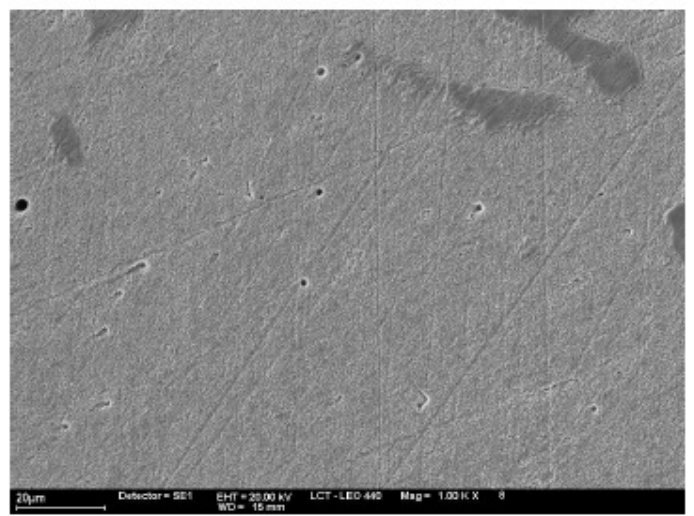

Figura 2.3. Aço 1008 atacado por $\mathrm{H}_{2} \mathrm{SO}_{4} 0.5$ mol. $\mathrm{L}^{-1}$ na presença de BTAH $10^{-3} \mathrm{M}-1000 \times$.

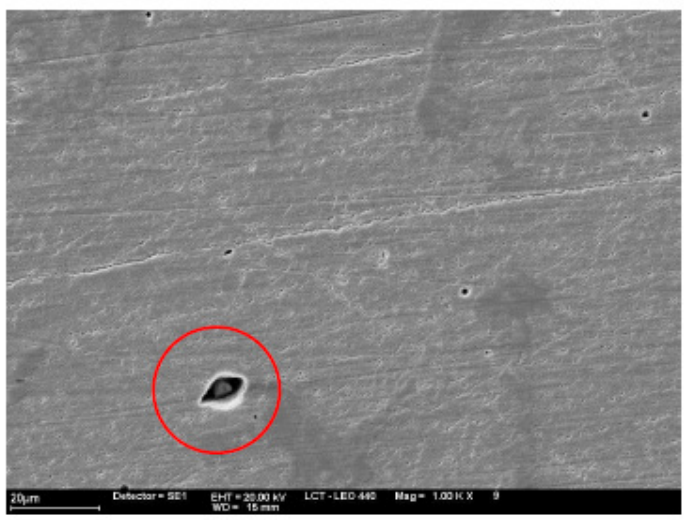

Figura 2.5. Aço 1008 atacado por $\mathrm{H}_{2} \mathrm{SO}_{4} 0.5$ Figura 2.6. Aço 1008 atacado por $\mathrm{H}_{2} \mathrm{SO}_{4} 0.5$ mol. $L^{-1}$ na presença de TTAH $10^{-2} \mathrm{M}-1000 \times$.

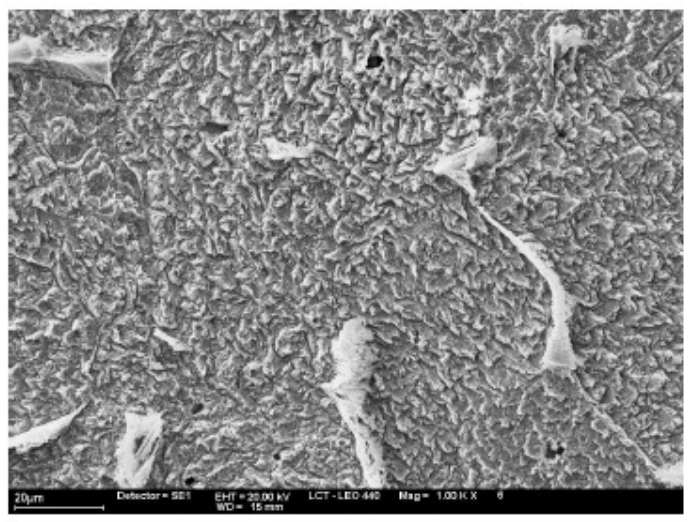

Figura 2.2. Aço 1008 atacado por $\mathrm{H}_{2} \mathrm{SO}_{4} 0.5$ mol. $L^{-1}$ na ausência de inibidores $-1000 \times$.

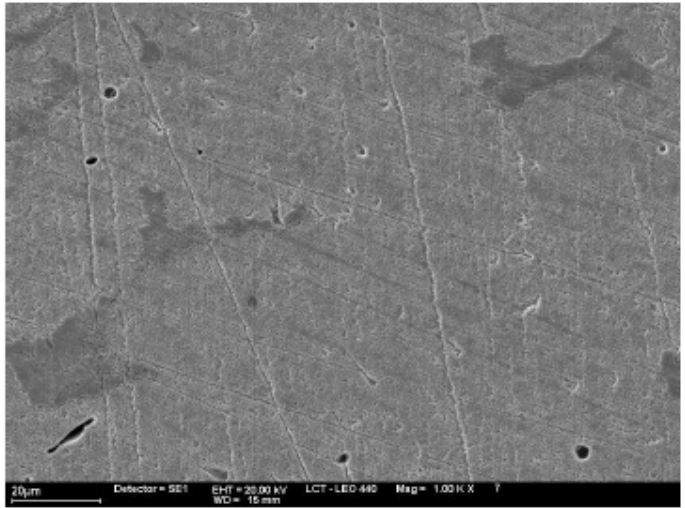

Figura 2.4. Aço 1008 atacado por $\mathrm{H}_{2} \mathrm{SO}_{4} 0.5$ mol. $L^{-1}$ na presença de BTAH $10^{-2} \mathrm{M}-1000 \times$.

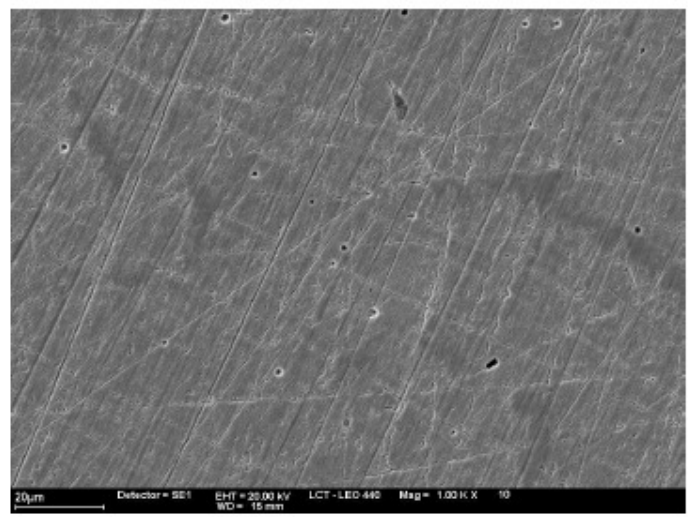

mol. $L^{-1}$ na presença de TTAH $10^{-2} \mathrm{M}-1000 \times$. 
As Fig. 2.1 e 2.2 apresentam um ataque generalizado onde se vêem ilhas de ferlita dentro de uma matriz tipicamente ferrítica. As ilhas ferríticas se encontram em relevo, mostrando que o ataque é muito maior interno sobre a fase ferrítica.

Nas Fig. 2.3 a 2.6 se constata a ação dos inibidores BTAH e TTAH, a ponto de as estruturas ferríticas e perlítica não serem reveladas. Vê-se, também, uma inibição maior a uma maior concentração. Os pontos de inclusão mostrados nestas micrografias indicam que os inibidores BTAH e TTAH não inibem o processo de dissolução das mesmas.

Nas Fig. 3.1 e 3.2 são apresentadas às análises quantitativas por EDS (dispersão de energia), do ponto de inclusão (2.1) e matriz da superfície do aço carbono 1008 (2.2).

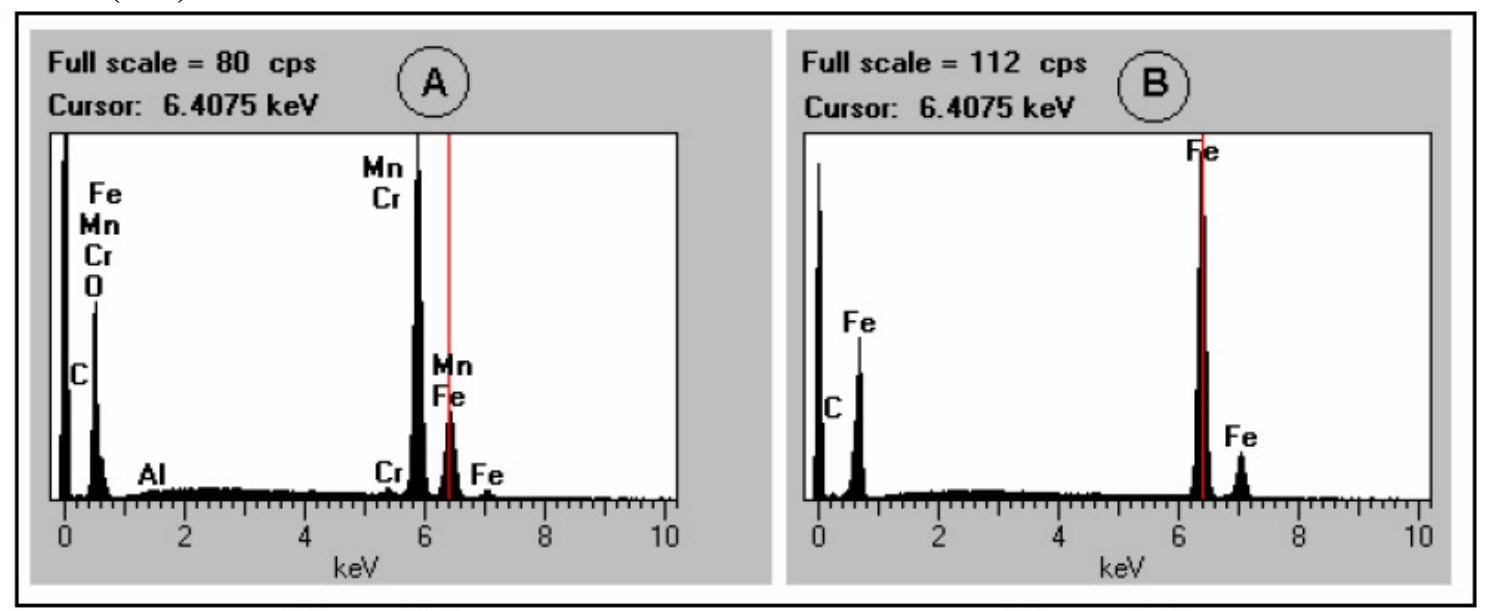

Figura 3.1

Figura 3.2

Figura 3. Análise quantitativa por EDS de ponto de inclusão ainda presente na matriz (3.1) e matriz da superfície do aço carbono 1008 (3.2).

Na Tabela 4 são apresentados quantitativamente os valores dos elementos constituintes da inclusão e da matriz, levando em conta a área superficial exposta do aço.

Tabela 4. Resultado da análise por EDS dos espectros da Fig. 2.

\begin{tabular}{|c|c|c|c|c|c|c|}
\hline \multirow{2}{*}{$\begin{array}{c}\text { Ponto de } \\
\text { análise }\end{array}$} & \multicolumn{6}{|c|}{ Percentual atômico dos elementos (\%) } \\
\cline { 2 - 7 } & $\mathrm{C}$ & $\mathrm{O}$ & $\mathrm{Al}$ & $\mathrm{Cr}$ & $\mathrm{Mn}$ & $\mathrm{Fe}$ \\
\hline A) inclusão & 0.05 & 51.2 & 0.30 & 0.62 & 39.9 & 7.86 \\
\hline B) matriz & 0.08 & -- & -- & -- & -- & 99.92 \\
\hline
\end{tabular}

\section{Curvas de polarização potenciostáticas}

Curvas de polarização potenciostática anódica

As curvas de polarização potenciostática anódicas para o aço carbono ABNT 1008 em meio de $\mathrm{H}_{2} \mathrm{SO}_{4} 0.5$ mol.L ${ }^{-1}$, contendo diferentes concentrações de BTAH, TTAH e misturas BTAH + TTAH, são apresentadas nas Fig. 4 a 7. 


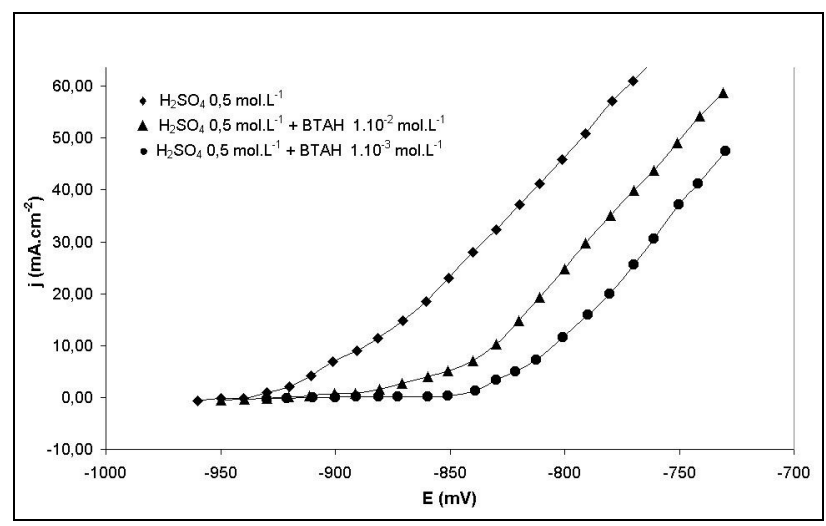

Figura 4. Curvas de polarização potenciostática anódica para o aço carbono ABNT 1008 em meio de: $(\bullet) \mathrm{H}_{2} \mathrm{SO}_{4} 0.5$ mol.L $\mathrm{L}^{-1}$; $\bullet \mathrm{H}_{2} \mathrm{SO}_{4} 0.5$ mol.L ${ }^{-1}+\mathrm{BTAH} 1 \times 10^{-3}$ mol.L ${ }^{1}$; (ム) $\mathrm{H}_{2} \mathrm{SO}_{4} 0.5$ mol.L $\mathrm{L}^{-1}+\mathrm{BTAH} 1 \times 10^{-2}$ mol.L $\mathrm{L}^{-1}$.

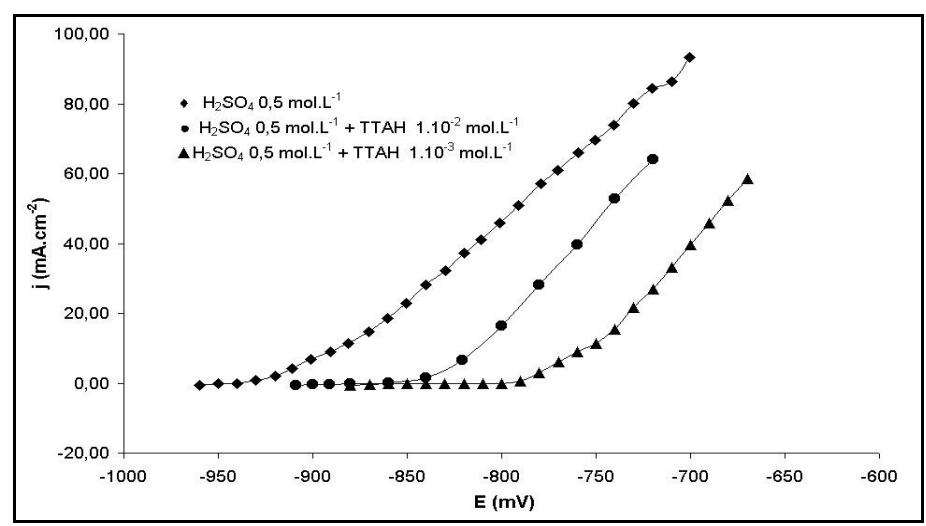

Figura 5. Curvas de polarização potenciostática anódica para o aço carbono ABNT 1008 em meio de: $(\bullet) \mathrm{H}_{2} \mathrm{SO}_{4} 0.5$ mol.L $\mathrm{L}^{-1}$; (ム) $\mathrm{H}_{2} \mathrm{SO}_{4} 0.5$ mol.L ${ }^{-1}+\mathrm{TTAH} 1 \times 10^{-3}$ mol.L $\mathrm{L}^{-}$ ; (•) $\mathrm{H}_{2} \mathrm{SO}_{4} 0.5 \mathrm{~mol} . \mathrm{L}^{-1}+\mathrm{TTAH} 1 \times 10^{-2} \mathrm{~mol} . \mathrm{L}^{-1}$.

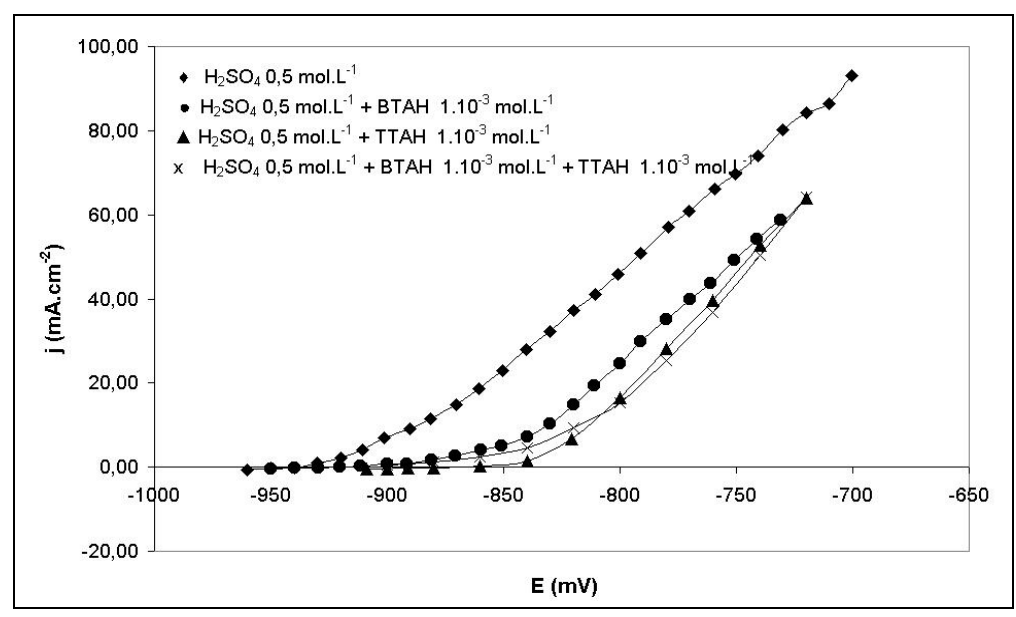

Figura 6. Curvas de polarização potenciostática anódica para o aço carbono ABNT 1008 em meio de: $(\bullet) \mathrm{H}_{2} \mathrm{SO}_{4} 0.5$ mol.L $\left.{ }^{-1} ; \bullet\right) \mathrm{H}_{2} \mathrm{SO}_{4} 0.5$ mol.L $\mathrm{L}^{-1}+\mathrm{BTAH} 1 \times 10^{-3}$

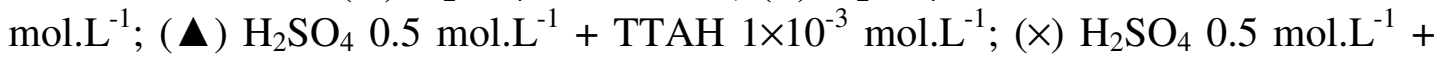
BTAH $1 \times 10^{-3}$ mol. $L^{-1}+$ TTAH $1.10^{-3}$ mol.L $L^{-1}$. 
Nas Fig. 4 e 5 verifica-se que o aumento da concentração do BTAH e TTAH diminui a densidade de corrente por toda extensão de potencial anódico estudada, indicando uma maior eficiência inibidora.

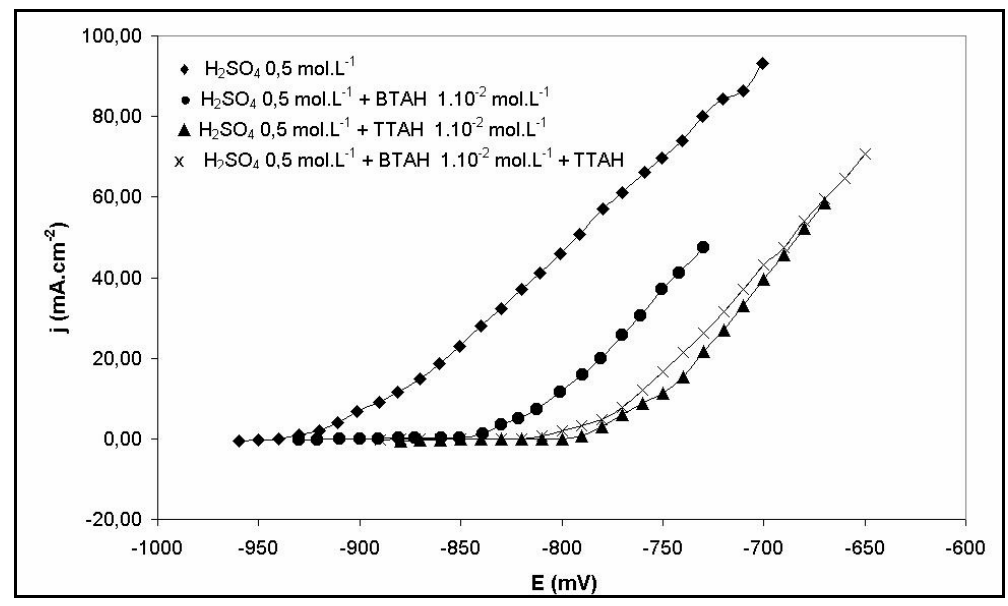

Figura 7. Curvas de polarização potenciostática anódica para o aço carbono ABNT 1008 em meio de: $(\bullet) \mathrm{H}_{2} \mathrm{SO}_{4} 0.5$ mol.L $\mathrm{L}^{-1} ;(\bullet) \mathrm{H}_{2} \mathrm{SO}_{4} 0.5$ mol.L $\mathrm{L}^{-1}+\mathrm{BTAH} 1 \times 10^{-2}$ mol.L ; ( $(\boldsymbol{\Delta}) \mathrm{H}_{2} \mathrm{SO}_{4} 0.5$ mol.L $\mathrm{L}^{-1}+\mathrm{TTAH} 1 \times 10^{-2} \mathrm{~mol} . \mathrm{L}^{-1}$; $(\times) \mathrm{H}_{2} \mathrm{SO}_{4} 0.5 \mathrm{~mol} . \mathrm{L}^{-1}+\mathrm{BTAH}$ $1 \times 10^{-2}$ mol. $L^{-1}+$ TTAH $1.10^{-2}$ mol. $L^{-1}$.

Na Fig. 6 se observa, para uma concentração de inibidor igual a $1 \times 10^{-3}$ mol.L ${ }^{-1}$, que o TTAH e a mistura BTAH + TTAH apresentam eficiência inibidora comparável e maior do que quando se emprega apenas BTAH.

Na Fig. 7 é observado o mesmo comportamento, para a concentração $1 \times 10^{-2}$ mol.L $L^{-1}$ de inibidor.

\section{Curvas de polarização potenciostática catódica}

As curvas de polarização potenciostáticas catódicas para o aço carbono ABNT 1008 em meio de $\mathrm{H}_{2} \mathrm{SO}_{4} 0.5$ mol.L ${ }^{-1}$ na ausência e presença de BTAH, TTAH e misturas BTAH + TTAH, são apresentadas nas Fig. 8 a 11.

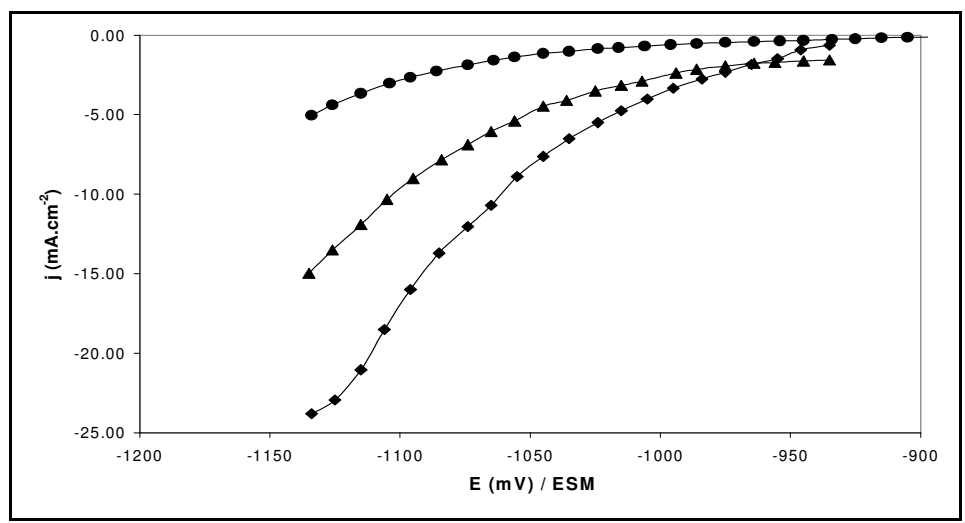

Figura 8. Curvas de polarização potenciostática catódica para o aço carbono ABNT 1008 em meio de: $(\bullet) \mathrm{H}_{2} \mathrm{SO}_{4} 0.5$ mol.L ${ }^{-1}$; $\bullet$ ) $\mathrm{H}_{2} \mathrm{SO}_{4} 0.5$ mol.L ${ }^{-1}+\mathrm{BTAH} 1 \times 10^{-3}$ mol.L ${ }^{-}$ ${ }^{1}$; ( $\left.\mathbf{\Delta}\right) \mathrm{H}_{2} \mathrm{SO}_{4} 0.5$ mol. $\mathrm{L}^{-1}+\mathrm{BTAH} 1 \times 10^{-2}$ mol.L $\mathrm{L}^{-1}$. 


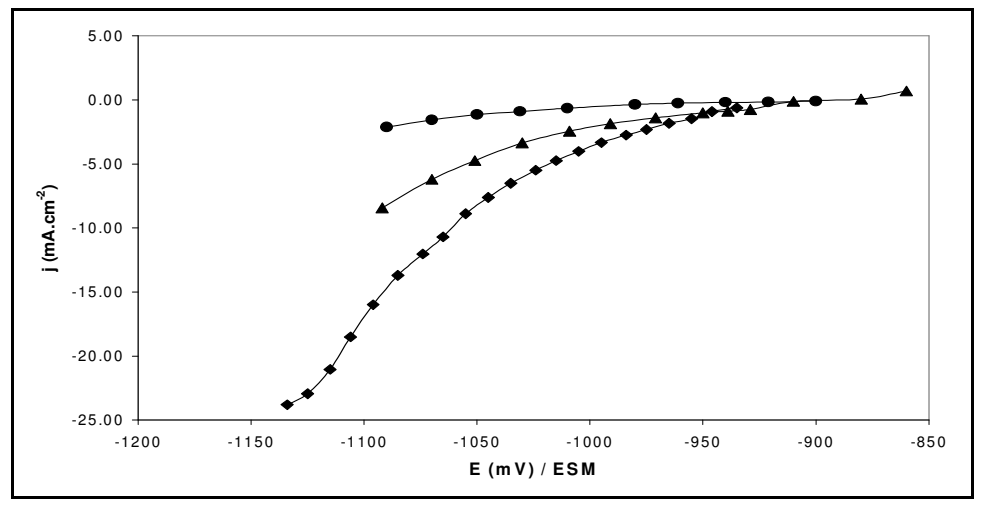

Figura 9. Curvas de polarização potenciostática catódica para o aço carbono ABNT 1008 em meio de: $(\bullet) \mathrm{H}_{2} \mathrm{SO}_{4} 0.5$ mol.L ${ }^{-1} ;(\bullet) \mathrm{H}_{2} \mathrm{SO}_{4} 0.5$ mol.L ${ }^{-1}+\mathrm{TTAH} 1 \times 10^{-3}$ mol.L $\mathrm{L}^{-1} ;(\boldsymbol{\Delta}) \mathrm{H}_{2} \mathrm{SO}_{4} 0.5$ mol.L $\mathrm{L}^{-1}+\mathrm{TTAH} 1 \times 10^{-2}$ mol.L $\mathrm{L}^{-1}$.

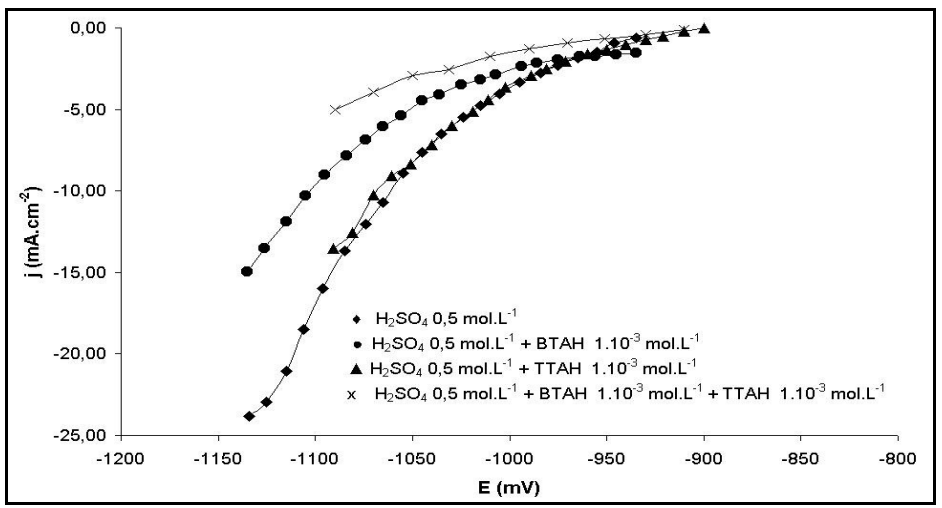

Figura 10. Curvas de polarização potenciostática catódica para o aço carbono ABNT 1008 em meio de: $(\bullet) \mathrm{H}_{2} \mathrm{SO}_{4} 0.5$ mol.L $\mathrm{L}^{-1}$; $\bullet \mathrm{H}_{2} \mathrm{SO}_{4} 0.5$ mol.L ${ }^{-1}+\mathrm{BTAH} 1 \times 10^{-3}$ mol.L ; ( $\mathbf{\Delta}) \mathrm{H}_{2} \mathrm{SO}_{4} 0.5 \mathrm{~mol} . \mathrm{L}^{-1}+$ TTAH $1 \times 10^{-3} \mathrm{~mol} . \mathrm{L}^{-1} ;(\times) \mathrm{H}_{2} \mathrm{SO}_{4} 0.5 \mathrm{~mol} . \mathrm{L}^{-1}+\mathrm{BTAH}$ $1 \times 10^{-3}$ mol. $\mathrm{L}^{-1}+$ TTAH $1 \times 10^{-3} \mathrm{~mol} \cdot \mathrm{L}^{-1}$.

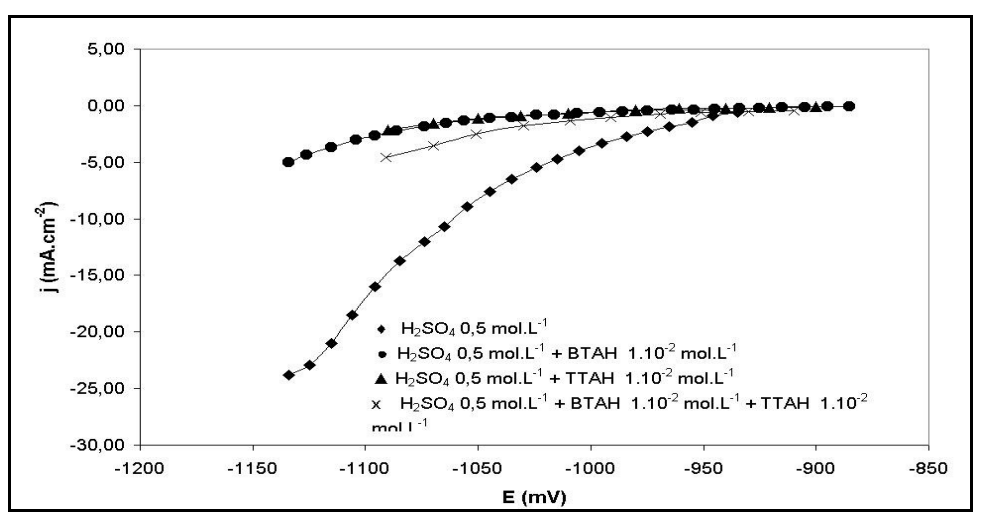

Figura 11. Curvas de polarização potenciostática catódica para o aço carbono ABNT 1008 em meio de: $(\bullet) \mathrm{H}_{2} \mathrm{SO}_{4} 0.5$ mol.L $\mathrm{L}^{-1}$; $\bullet \mathrm{H}_{2} \mathrm{SO}_{4} 0.5$ mol. $\mathrm{L}^{-1}+\mathrm{BTAH} 1 \times 10^{-2}$ mol.L

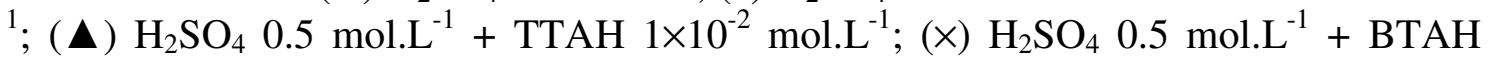
$1 \times 10^{-2}$ mol. $\mathrm{L}^{-1}+$ TTAH $1 \times 10^{-2}$ mol. $\mathrm{L}^{-1}$.

Nota-se nas Fig. 8 e 9 que quanto maior a concentração de BTAH ou TTAH na solução de $\mathrm{H}_{2} \mathrm{SO}_{4} 0.5$ mol.L ${ }^{-1}$, menor a densidade de corrente registrada, sugerindo uma maior ação inibidora. 
Na Fig. 10 se verifica que a densidade de corrente em toda a região de potencial catódico estudado é menor para o sistema com a mistura BTAH $1 \times 10^{-3} \mathrm{~mol}^{-\mathrm{L}^{-1}+}$ TTAH $1 \times 10^{-3}$ mol. $\mathrm{L}^{-1}$, em relação ao sistema apenas contendo BTAH $1.10^{-3}$ mol.L ${ }^{-1}$ ou TTAH.

Na Fig. 11 nota-se que na presença de compostos orgânicos a uma concentração de $1 \times 10^{-2}$ mol. $\mathrm{L}^{-1}$ ou na mistura dos mesmos, a densidade de corrente é menor em toda região de potencial catódico estudada, quando se compara com o sistema sem inibidor, mas a mistura BTAH + TTAH inibe menos do que os inibidores separadamente. Curvas de Tafel para a polarização catódica em quatro meios estudados $\left(\mathrm{H}_{2} \mathrm{SO}_{4}\right.$ 0.5 mol.L ${ }^{-1}, \mathrm{H}_{2} \mathrm{SO}_{4} 0.5$ mol.L $\mathrm{L}^{-1}+\mathrm{BTAH} 1 \times 10^{-3}$ mol.L ${ }^{-1}$, $\mathrm{H}_{2} \mathrm{SO}_{4} 0.5$ mol.L $\mathrm{L}^{-1}+$ TTAH $1 \times 10^{-3}$ mol.L $\mathrm{L}^{-1}$ e $\mathrm{H}_{2} \mathrm{SO}_{4} 0.5$ mol. $\mathrm{L}^{-1}+\mathrm{BTAH} 1 \times 10^{-3}$ mol. $\mathrm{L}^{-1}+$ TTAH $1 \times 10^{-3}$ mol. $\mathrm{L}^{-1}$ ), obtidas a uma sobretensão superior a $100 \mathrm{mV}$ em relação ao $\mathrm{E}_{\text {corr }}$ mostraram dentro de uma precisão de $10 \%$ igual ao coeficiente angular. Estes resultados aliados aos gravimétricos sugerem que estes inibidores atuem por bloqueio de superfície e que a interação entre eles só é destrutiva, quando $\theta \rightarrow 1$. Em baixa concentração, eles se adsorvem independentemente sem afetar o mecanismo dos processos catódicos. Esta análise é apenas qualitativa, uma vez que o meio é naturalmente aerado, com a presença de mais de um processo de redução.

\section{Curvas cronoamperométricas}

Na Fig. 12 são apresentadas as curvas cronoamperométricas para o aço carbono 1008 nos diferentes meios estudados, a uma sobretensão anódica de $50 \mathrm{mV}$ a partir do $\mathrm{E}_{\text {corr. }}$ Os resultados mostram que a cinética de adsorção dos inibidores é rápida e que o valor estacionário de corrente é obtido em um intervalo menor de tempo quanto maior a concentração de BTAH ou TTAH. Observa-se ainda que o TTAH se mostra melhor inibidor do que o BTAH.

\section{Diagramas de impedância eletroquímica}

Os resultados de espectroscopia de impedância eletroquímica (EIE) são apresentados na Fig. 13, para todos os meios de $\mathrm{H}_{2} \mathrm{SO}_{4} 0.5$ mol.L $\mathrm{L}^{-1}$ sem e com BTAH, TTAH e BTAH + TTAH a concentração de $1 \times 10^{-3}$ mol.L $L^{-1}$ para $\mathrm{E}=$ $900 \mathrm{mV} / \mathrm{ESM}$.

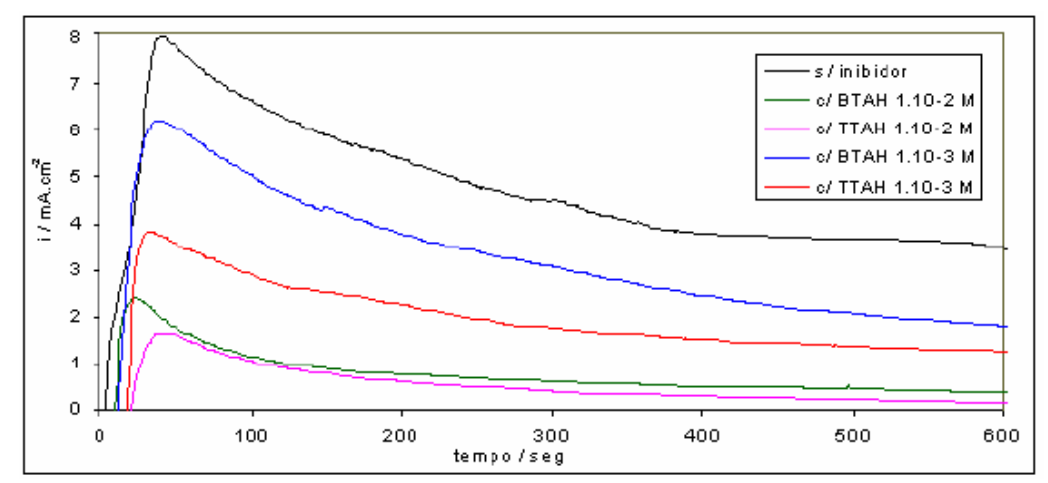

Figura 12. Cronoamperogramas a uma sobretensão anódica de $50 \mathrm{mV}$ a partir do $\mathrm{E}_{\text {corr }}$ para o aço carbono 1008 em meio de ácido sulfúrico 0.5 mol. $\mathrm{L}^{-1}$ na ausência e presença dos inibidores. 


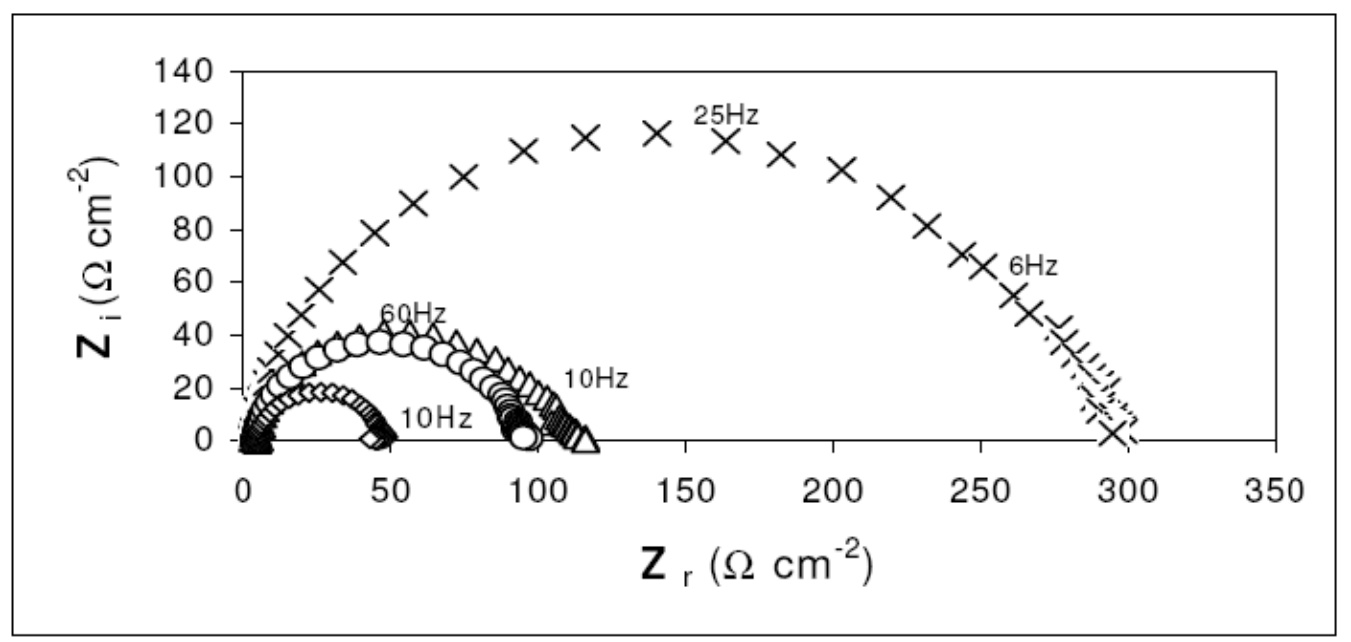

Figura 13. Diagramas de impedância eletroquímica para o aço carbono $1008 \mathrm{em}$ meio de: $(\diamond) \mathrm{H}_{2} \mathrm{SO}_{4} 0.5$ mol.L $\mathrm{L}^{-1}$; $(\circ) \mathrm{H}_{2} \mathrm{SO}_{4} 0.5$ mol.L $\mathrm{L}^{-1}+\mathrm{BTAH} 1 \times 10^{-3}$ mol.L $\mathrm{L}^{-1} ;(\Delta)$ $\mathrm{H}_{2} \mathrm{SO}_{4} 0.5$ mol.L ${ }^{-1}+$ TTAH $1 \times 10^{-3}$ mol.L $\mathrm{L}^{-1}$ ( $\times$ ) $\mathrm{H}_{2} \mathrm{SO}_{4} 0.5$ mol. $\mathrm{L}^{-1}+$ BTAH $1 \times 10^{-3}$ mol. $\mathrm{L}^{-1}+\mathrm{TTAH} 1 \times 10^{-3} \mathrm{~mol} \cdot \mathrm{L}^{-1}$.

Os diagramas de EIE da Fig. 13 mostram que a resistência de polarização (Rp), aumenta na presença de inibidores. Ressalta-se o fato de que o maior valor de Rp foi registrado para a mistura BTAH $1 \times 10^{-3}$ mol.L ${ }^{-1}+$ TTAH $1 \times 10^{-3}$ mol.L ${ }^{-1}$, confirmando o efeito sinérgico inibidor observado nas medidas gravimétricas e nas curvas de polarização potenciostáticas catódicas do aço carbono ABNT 1008 em meio $\mathrm{H}_{2} \mathrm{SO}_{4} 0.5$ mol.L $\mathrm{L}^{-1}$.

\section{Conclusões}

1) $\mathrm{O}$ aço carbono 1008 estudado apresentou um número médio de inclusões correspondente a $1.6 \%$ de área superficial analisada.

2) O benzotriazol (BTAH) e o tolitriazol (TTAH) mostram-se inibidores de corrosão para o aço carbono ABNT 1008 em meio de $\mathrm{H}_{2} \mathrm{SO}_{4} 0.5$ mol. $\mathrm{L}^{-1}$.

3) A máxima eficiência observada, igual a $(96 \pm 2) \%$, corresponde a uma concentração igual a $1 \times 10^{-2}$ mol.L $\mathrm{L}^{-1}$ de BTAH e de TTAH, respectivamente. O grau de recobrimento máximo coincide com outros dados da literatura e com a área superficial livre de inclusões.

4) O TTAH se mostrou melhor inibidor para o processo de oxidação do aço em todo o intervalo de potencial estudado.

5) A mistura BTAH $1 \times 10^{-3}$ mol. $\mathrm{L}^{-1}+$ TTAH $1 \times 10^{-3}$ mol. $\mathrm{L}^{-1}$ demonstrou um efeito sinérgico na inibição da corrosão do aço 1008 em meio de $\mathrm{H}_{2} \mathrm{SO}_{4} 0.5$ mol.L $\mathrm{L}^{-1}$, no potencial de corrosão (por ensaios gravimétricos) e para $\mathrm{o}$ processo catódico em toda a região de potencial estudada neste trabalho.

6) Valores de $R_{p}$, obtidas por EIE, a um potencial igual a $-900 \mathrm{mV} / \mathrm{ESM}$, mais positivo do que os valores de $\mathrm{E}_{\text {corr }}$ das interfases estudadas, também mostraram que a mistura BTAH $1 \times 10^{-3}$ mol.L ${ }^{-1}+$ TTAH $1 \times 10^{-3}$ mol. $\mathrm{L}^{-1}$ apresentou melhor eficiência inibidora que os inibidores separadamente. 


\section{Agradecimentos:}

À fundação Araucária (PR) e a Finep pelo auxílio e apoio a esta pesquisa. Ao CNPq pela concessão das bolsas de mestrado.

\section{Benzotriazol e Tolitriazol como Inibidores de Corrosão para o Aço Carbono 1008 em Ácido Sulfúrico}

\section{Resumo}

Neste trabalho as características de corrosão e inibição do aço carbono 1008 em meio de $\mathrm{H}_{2} \mathrm{SO}_{4} \quad 0.5$ mol.L $\mathrm{L}^{-1}$ foram investigadas por métodos físicos e eletroquímicos. Nos estudos de inibição da corrosão empregaram-se compostos orgânicos contendo heteroátomo de $\mathrm{N}$, como o benzotriazol (BTAH), tolitriazol (TTAH) e misturas BTAH + TTAH. As técnicas empregadas nos estudos foram: medidas de potencial de circuito aberto, curvas de polarização potenciodinâmica anódica, curvas de cronoamperometria, diagramas de impedância, perdas de massa e análise óptica de superfícies. Os resultados de polarização anódica mostraram que o aumento da concentração do BTAH e TTAH diminui a densidade de corrente por toda extensão de potencial estudado; estes resultados, associados aos gravimétricos, sugerem que estes inibidores atuem por bloqueio de superfície e que a interação entre eles só é destrutiva quando a eficiência inibidora é próxima de $100 \%$, demonstrando que em baixa concentração, o BTAH e o TTAH se adsorvem independentemente sem afetar o mecanismo dos processos catódicos. As curvas de polarização anódica mostram uma atuação inibidora acentuada para o TTAH, em todo o intervalo de potencial estudado; entretanto, os ensaios gravimétricos e os ensaios eletroquímicos mostraram um efeito sinérgico em relação à eficiência inibidora para a mistura de BTAH $1 \times 10^{-3}$ mol. $\mathrm{L}^{-1}+$ TTAH $1 \times 10^{-3} \mathrm{~mol} . \mathrm{L}^{-1}$.

Palavras-chave: aço carbono 1008, corrosão, inibidor, ácido sulfúrico, benzotriazol, tolitriazol.

\section{Referências}

1. G. Trabanelli, Corrosion Mechanisms, (F. Mansfeld) New York, Marcel Dekker (1987).

2. P. Spinelli and G. Hoxha, "Evaluation of Armco iron corrosion rate by impedance techniques", $9^{\text {th }}$ European Congress on Corrosion, Utrecht, The Netherlands, 2/6 oct., p. FU-15 (1989).

3. S. Brinic, Z. Grubac, R. Babic and Metikos-Hukovic, "Study of the thiourea adsorption on iron in acid solution", Proceedings of the $8^{\text {th }}$ European Symposium on Corrosion Inhibitors (7SEIC), Ann. Univ. Ferrara, v. 1, p. 197 (1995).

4. P.R.P. Rodrigues, "O benzotriazol como inibidor de corrosão para o ferro e ligas ferrosas em meio de ácido sulfúrico", Tese de Doutorado, IQUSP São Paulo, SP (1997).

5. M.T. Cunha, "Estudo comparativo dos inibidores benzotriazol e tolitriazol na oxidação de materiais ferrosos em meio de ácido sulfúrico", Dissertação de Mestrado, IQUSP - São Paulo, SP (2003). 
6. I.V.S. Santos, "Filme de Benzotriazol sobre Materiais Ferrosos: Formação, Resistência, Efeito de Aditivos e do Transporte de Massa", Dissertação de Mestrado, IQUSP - São Paulo, SP (2003).

7. $\quad$ K. Nobe, N. Elkadar, Corrosion 37 (1981) 4.

8. $\quad$ K. Nobe, N. Elkadar, Corrosion 36 (1981) 5.

9. K. Nobe, N. Elkadar, Corrosion 32 (1976).

10. G.K. Gomma, Materials Chemistry and Physica 56 (1998).

11. M.T. Cunha, P.R.P. Rodrigues, S.M.L. Agostinho, "Construção de um potenciostato com interface de baixo custo para medidas eletroquímicas", Anais do XVIII Simpósio Brasileiro de Eletroquímica e Eletroanalítica, Araraquara, São Paulo (2000). 